\title{
Psychosocial profile of the patients with inflammatory bowel disease
}

\author{
TIBERIA ILIAS $^{1}$, SIMONA BUNGAU ${ }^{2}$, DELIA MIRELA TIT ${ }^{2}$, DANIELA MAGHIAR $^{3}$, CRISTIAN HOCOPAN $^{1}$, \\ ROXANA BRATA $^{1}$, OVIDIU GABRIEL BRATU ${ }^{4}$, NICOLETA NEGRUT ${ }^{5}$, \\ CAMELIA DIACONU $^{6,7}$ and OVIDIU FRATILA ${ }^{1}$
}

\author{
${ }^{1}$ Department of Medical Disciplines, Faculty of Medicine and Pharmacy, University of Oradea, 410073 Oradea; \\ ${ }^{2}$ Department of Pharmacy, Faculty of Medicine and Pharmacy, University of Oradea, 410028 Oradea; \\ ${ }^{3}$ Emergency Clinical County Hospital, 410169 Oradea; ${ }^{4}$ Clinical Department 3, \\ 'Carol Davila' University of Medicine and Pharmacy, 050474 Bucharest; \\ ${ }^{5}$ Department of Psycho-Neuroscience and Recovery, Faculty of Medicine and Pharmacy, \\ University of Oradea, 410073 Oradea; ${ }^{6}$ Department 5, 'Carol Davila' University of Medicine and Pharmacy, \\ 050474 Bucharest; ${ }^{7}$ Internal Medicine Clinic, Clinical Emergency Hospital of Bucharest, 041914 Bucharest, Romania
}

Received January 29, 2020; Accepted February 28, 2020

DOI: 10.3892/etm.2020.8816

\begin{abstract}
Inflammatory bowel disease (IBD) is a chronic, disabling entity of unknown aetiology, with negative impact on the patient's life, including psychological patterns. This study assessed multiple psychosocial factors (satisfaction with life, coping mechanisms, emotional profile, mental recognition of the disease and cognition schemes related to patients' demographic characteristics, clinical picture, form and duration of the disease, therapeutic plans) in IBD patients vs. a healthy group. This non-interventional study comprised 60 participants who attended for medical advice/check-up as an ambulatory visit or during hospitalization. The patients completed questionnaires after receiving explanations from the psychologist. Statistical analyses (Kolmogorov-Smirnov test, Independent-Samples t-test, One-Way ANOVA and Post Hoc Multiple Comparisons) were conducted using IMB for the Social Sciences (SPSS), version 20 (P $\leq 0.05)$. IBD patients (G1) are more hostile when compared to the healthy group (G2). Those who experience abdominal pain are more likely to use active coping mechanisms and those who experience fatigue are more likely to use acceptance, emotional venting, behavioural disengagement and mental disengagement. G1 have higher levels of others-downing vs. G2. Regarding negative emotions, IBD patients generally experience more negative emotions compared to healthy participants (who have higher
\end{abstract}

Correspondence to: Professor Delia Mirela Tit, Department of Pharmacy, Faculty of Medicine and Pharmacy, University of Oradea, 1 December Square 10, 410028 Oradea, Romania

E-mail: dtit@uoradea.ro

Key words: inflammatory bowel disease, psychosocial factors, quality of life, psychological patterns, questionnaires levels of life satisfaction). Regarding the perception of illness, there are no differences between patients in terms of illness coherence, personal or treatment control, consequences, timeline, or emotional representations. Results indicate that psychological factors and different characteristics of IBD patients play a relevant role in the way these patients deal with their disease.

\section{Introduction}

Inflammatory bowel disease (IBD) is a chronic, disabling entity of unknown aetiology, including usually ulcerative colitis (UC) and Crohn's disease (CD). These two idiopathic diseases are affecting the gastrointestinal tract, being characterised by periods of activity/remission. CD can affect all of the digestive tract, whereas UC is localised only to the rectum and large bowl. Regarding symptoms, patients with UC typically complain of bloody stool and lower crampy abdominal pain, whereas patients with CD usually present fatigue, weight loss, chronic abdominal pain, diarrhoea. In IBD with long-standing course, complications with different prevalence including malabsorption/malnutrition, strictures, dysplasia and cancer. Unfortunately, the prevalence of IBD has increased during the last decade (1). Studies report the highest prevalence values in Europe (Norway: UC 505/100,000; Germany: CD 322/100,000) (2).

In Romania, there is scarce reported data regarding the epidemiology of IBD: an incidence of 0.97/100,000 and $0.50 / 100,000$ for UC and CD, respectively, and a prevalence of 2.42/100,000 and 1.51/100,000 for UC and CD (3). Another study estimated the incidence and prevalence of CD in South Romania, between 2005-2009 at 0.49/100,000 and $1.88 / 100,000$ inhabitants respectively. Regarding temporal tendency there was an increase in both parameters in 2009 by 32 and $69 \%$, respectively, as compared to 2005 . When it comes to predicting trends, the above-mentioned study anticipated a 
relatively stable incidence of $\mathrm{CD}$, but an upward movement of the prevalence between 2010-2014 (4).

Due to its long-standing course, frequent need and dependency of medical care, high costs of therapy and dietary restrictions, difficult mingling in society, fear of malignant transformation, IBD negatively impacts on many aspects of the patient's life, including psychological patterns. Though formal psychotherapy is not a mainstay tool in the primary treatment of IBD, it is to be assumed it might be of great importance in helping the patient coping with a chronic and possible debilitating disease.

In 2017, the American Gastroenterological Association assigned a task force to perform a large-scale review from the literature in order to obtain valuable information of how gastroenterologists should integrate psychosocial aspects of IBD into their daily practice, to provide the best care for the wellbeing of their patients. Thus, this study supports the conclusion that a combined, integrated approach (medical and psychological) will be of substantial value to IBD patients, and we should rely on screening and interventional methods of the psychosocial factors, leading to a patient-focused care (5).

Our research intended to assess multiple psychosocial factors (satisfaction with life, coping mechanisms, emotional profile, mental recognition of the disease and cognition schemes related to their demographic characteristics, clinical picture, form and duration of the disease, therapeutic plans) in IBD patients by comparison with a healthy witness group.

\section{Patients and methods}

Study design. A non-interventional study was conducted between 01.01.2018-31.01.2019. Sixty patients were included (during an ambulatory visit received as out-patients for medical advice/check-up at Medical Office Gastroenterology Dr Fratila, located in Oradea, Romania, or during hospitalization at 1st Medical Clinic, County Emergency Clinical Hospital (Oradea, Romania). After obtaining the informed consent from each participant, two 2 groups were studied: G1, 30 previously diagnosed IBD patients and G2 (control group), 30 healthy individuals (no history of chronic disease), with similar demographic characteristics (Table I).

The research was performed according the WMA Declaration of Ethics Helsinki Medical Research Involving Human Principles for Subjects and was approved by the Ethics Committee of the Clinical County Emergency Hospital of Oradea (Romania). All the subjects signed a written consent giving their acceptance to participate in the study.

Variables and questionnaires. The method of the study was the use of self-administered questionnaires, after the psychologist involved in the study explained the rules of completion. These questionnaires are as follows:

The Buss-Perry Aggression Questionnaire (TAQ, The Aggression Questionnaire) was used, as it distinguishes between the 4 dimensions of aggression: physical, verbal, anger and hostility. The questionnaire has 29 statements that are evaluated on a 5-point Likert scale (6), varying from 1 , 'extremely uncharacteristic of me' to 5, 'extremely characteristic of me'. Higher scores represent higher levels of aggression.
COPE, the multidimensional coping inventory was used to assess the ways in which the participants respond to stress. The items are scored on a 4-point Likert scale, with scores ranging from 1 ('I don't do this at all') to 4 ('I usually do this'). Items are summed to produce scale scores, with higher scores reflecting greater use of a particular coping strategy. The scale has 13 subscales, with 5 of them measuring problem focused strategies, that aim to reduce or eliminate the direct cause of stress (active coping, planning, suppression of competing activities, restraint coping and seeking social support for instrumental reasons), the further five subscales evaluating emotion-focused strategies (seeking of social support for emotional reason, positive reinterpretation, acceptance, denial and turning to religion). The following 3 subscales, considered by the authors less useful are labelled focus on and venting of emotions (acknowledging and expressing your feelings), behavioural and mental disengagement (7). The last item is used to assess the use of alcohol and drugs.

Unconditional Self-Acceptance Questionnaire (USAQ) evaluates the unconditional self-acceptance; it uses a 7-point Likert scale (1, 'almost always not true' to 7, 'almost always true') to assess an individual's ability to maintain a positive, global sense of self, among any shortcomings and/or failures reflecting an individual's capacity to embrace both their strengths and weaknesses, and often serves as an indication of personal satisfaction and happiness (8-10).

The General Attitudes and Beliefs Scale-Short Version (GABS-SV) (11) was used as a measurement of irrational cognitions. The items of this scale are derived from current REBT theory (12) and they assess both irrational cognitive processes (self-downing, others-downing) and needs (achievement, approval, comfort and demands for fairness), which are considered to lead to dysfunctional emotions. A global score of irrationality can be obtained by summing the afore mentioned subscales. Participants rate each item on a 5-point Likert scale to indicate level of agreement (1 meaning, 'strongly disagree' and 5, 'strongly agree'). The scale also includes a rationality subscale.

The Profile of Affective Distress (PAD) was used to assess the functional/dysfunctional patients' emotions. The scale generates scores for global distress, negative emotions, but more specifically for concern (functional)/anxiety (dysfunctional), sadness (functional)/depression (dysfunctional) and positive emotions. The participants evaluate the degree to which they felt the 39 items (adjectives describing emotions) during the last 2 weeks on a scale form 1, 'not at all' to 5, 'a lot' (13).

Satisfaction with Life Scale (SLS) (14) is a 5 item scale used to assess the global cognitive judgements of life satisfaction on a 7-point scale (1, 'strongly disagree', 7, 'strongly agree'), with higher scores representing higher levels of satisfaction.

The Illness Perception Questionnaire-Revised (IPQ-R) was used to assess the patients' beliefs concerning their disease in terms of timeline (chronic vs. acute), cyclicality, controllability of the disease/treatment, emotional representation, illness coherence (comprehension and expectancies regarding the disease). The identity of the disease is also assessed by evaluating the presence/absence of symptoms typically associated with the disease (15). Therefore, a modified list of symptoms was used to fit the clinical profile of IBD (abdominal pain, diarrhoea, bloating, anal bleeding, fatigue, nausea, arthralgia, 
Table I. Demographic characteristics of G1 and G2.

\begin{tabular}{lcc}
\hline Sample characteristics & $\mathrm{G} 1$ & $\mathrm{G} 2$ \\
\hline Variables & & \\
$\mathrm{n}$ & 30 & 30 \\
Age-mean, years & 44.5 & 33.7 \\
SD; range & $12.6 ; 26-71$ & $10.47 ; 20-57$ \\
Sex, $\mathrm{n}(\%)$ & & \\
$\quad$ Female & $16(54)$ & $18(60)$ \\
Environment n, (\%) & & \\
Urban & $27(90)$ & $27(90)$ \\
Rural & $3(10)$ & $3(10)$ \\
Type of IBD, n (\%) & & \\
CD & $14(23.3)$ & \\
UC & $16(26.7)$ & \\
IBD duration, mean years & & \\
$<7$ & 20 & \\
$>7$ & 10 & \\
Therapy n, (\%) & & \\
Immunosuppressant & $12(40)$ & \\
Biological & $22(73)$ & \\
\hline
\end{tabular}

G1, IBD patients; G2, healthy group; IBD, inflammatory bowel disease; $\mathrm{UC}$, ulcerative colitis; $\mathrm{CD}$, Crohn's disease.

fever, weight loss and dizziness). The questionnaire also uses a list of 18 possible culprits for their disease and encourages the participants to identify the first three most likely elements. Items describing symptoms are evaluated on a binary 'yes/no' basis, while the rest of the items are evaluated on a 5-point scale, from 1, 'strongly disagree' to 5 , 'strongly agree'. High scores on the identity, timeline, consequences, and cyclical dimensions represent strong beliefs about the number of symptoms attributed to the illness, the chronicity of the disease, the negative outcomes, and its cyclical nature, whereas high scores on the personal and treatment control subscales, represent positive beliefs about the controllability over the disease. High scores on coherence represent a personal understanding of the condition (15).

First test filled in by G2 was COPE, followed by TAQ, USAQ, GABS-SV, PAD and the Satisfaction with Life Scale. G1 patients started with IPQ-R and continued in the above-mentioned order.

Statistical analysis. Statistical analyses were performed using IMB for the Social Sciences (SPSS), version 20, $P \leq 0.05$. To assess the assumption of normality of the gathered data, the Kolmogorov-Smirnov (K-S) test was used (as a goodness of fit test). Parametric tests were used for further analyses: Independent-samples t-test was used to compare health status, severity of the disease and clinical features; One-Way ANOVA was used to compare our subjects depending on the type of the disease and years since diagnosis; least significance difference (LSD) test was used for Post Hoc Multiple Comparisons where significant results were obtained.

\section{Results}

The significant outcomes of the tests and the levels of statistical significance G1 vs. G2 are showed in Table II.

Regarding the differences between patients with IBD, the statistically significant results are presented in Table III.

$T A Q$. There are no significant differences between participants with regards to the level of verbal/physical aggressiveness or anger depending on health status, type of disease, severity of disease and presence/absence of symptoms. IBD patients (G1) are more hostile when compared to $\mathrm{G} 2(\mathrm{P}=0.03<0.05)$; especially the patients with moderate forms of IBD are significantly more hostile than healthy persons $(\mathrm{P}=0.005<0.01)$. Also, patients with long-standing disease ( $>7$ years) have higher levels of hostility than the $\mathrm{G} 2(\mathrm{P}=0.01<0.05 ; \mathrm{P}=0.005<0.01)$.

COPE. No significant differences were obtained among coping mechanisms, depending on health status (G1 vs. G2), even when differentiating the two type of disease (comparison between the healthy individuals, patients with $\mathrm{CD}$ or $\mathrm{UC}$ ). Evaluations between patients depending on the duration of the disease revealed no significant differences, either. However, when taking into account the severity of the disease, patients with moderate severity use emotional venting more than patients with severe forms of IBD $(\mathrm{P}=0.03<0.05)$. Patients with moderate forms of the disease also use positive reinterpretation more, compared to those with severe forms $(\mathrm{P}=0.04<0.05)$. G2 patients use restraint coping more than patients with severe IBD $(\mathrm{P}=0.03<0.05)$.

Patients who experience abdominal pain are more likely to use active coping mechanisms $(\mathrm{P}=0.03<0.05)$. They also seek more social support for instrumental reasons $(\mathrm{P}=0.03<0.05)$ and use positive reinterpretation more $(\mathrm{P}=0.02<0.05)$. Patients who experience fatigue are more likely to use acceptance ( $\mathrm{P}=0.02<0.05)$, emotional venting $(\mathrm{P}=0.03<0.05)$ behavioural disengagement $(\mathrm{P}=0.01<0.05)$ and mental disengagement $(\mathrm{P}=0.001<0.01)$ compared to patients who do not experience this symptom. Patients with arthralgia are more likely to use emotional venting than those who do not experience this symptom $(\mathrm{P}=0.005<0.01)$.

USAQ . No significant differences were obtained between G1 and $\mathrm{G} 2$, nor regarding assessment of duration of the disease, type of illness, presence of symptoms such as abdominal pain, fatigue and arthralgia with respect to the unconditional self-acceptance. However, patients with moderate forms of the disease have a higher level of unconditional self-acceptance compared to patients with severe forms $(\mathrm{P}=0.01<0.05)$ and with those from $\mathrm{G} 2(\mathrm{P}=0.01<0.05)$.

GABS. Patients in G1 have higher levels of others-downing when compared to $\mathrm{G} 2(\mathrm{P}=0.01<0.05)$. More specifically, patients with UC tend to judge others more (others-downing), when compared to healthy persons $(\mathrm{P}=0.02<0.05)$. No significant differences were found between patients with $\mathrm{UC}$ and $\mathrm{CD}$, nor when comparing patients with $\mathrm{CD}$ to $\mathrm{G} 2$. There are no differences between the participants regarding duration of the disease. When considering the severity of the disease, patients with moderate forms are more judgemental (others-downing) compared to $\mathrm{G} 2(\mathrm{P}=0.009<0.01)$. No other significant results 
Table II. IBD patients vs. healthy participants (G1 vs. G2).

\begin{tabular}{|c|c|c|c|}
\hline Questionnaire & $\begin{array}{c}\text { Investigated } \\
\text { characteristic/subscale }\end{array}$ & Compared groups & Significance \\
\hline \multirow[t]{3}{*}{ TAQ } & \multirow[t]{3}{*}{ Hostility } & IBD $>$ healthy & 0.03 \\
\hline & & Moderate $>$ healthy & 0.005 \\
\hline & & More than 7 years since diagnosis $>$ healthy & 0.005 \\
\hline COPE & Restraint coping & Healthy individuals $>$ IBD patients & 0.03 \\
\hline USAQ & Unconditional self-acceptance & Moderate $>$ healthy & 0.01 \\
\hline \multirow[t]{4}{*}{ GABS } & \multirow[t]{3}{*}{ Others-downing } & Healthy $>$ severe & 0.01 \\
\hline & & IBD $>$ healthy & 0.01 \\
\hline & & $\mathrm{UC}>$ healthy & 0.02 \\
\hline & Rationality & Moderate $>$ healthy & 0.009 \\
\hline \multirow[t]{15}{*}{ PAD } & \multirow[t]{2}{*}{ Negative emotions } & IBD $>$ healthy & 0.02 \\
\hline & & Moderate $>$ healthy & 0.04 \\
\hline & \multirow[t]{6}{*}{ Positive emotions } & Healthy > IBD & 0.008 \\
\hline & & Healthy $>$ Crohn's disease & 0.01 \\
\hline & & Healthy $<7$ years since diagnosis & 0.01 \\
\hline & & Healthy $>7$ years since diagnosis & 0.03 \\
\hline & & Healthy $>$ moderate & 0.04 \\
\hline & & Healthy $>$ severe & 0.04 \\
\hline & Functional negative emotions & IBD $>$ healthy & 0.05 \\
\hline & \multirow[t]{5}{*}{ Anxiety } & IBD $>$ healthy & 0.01 \\
\hline & & $\mathrm{UC}>$ healthy & 0.006 \\
\hline & & Less than 7 years since diagnosis $>$ healthy & 0.04 \\
\hline & & More than 7 years since diagnosis $>$ healthy & 0.05 \\
\hline & & Moderate $>$ healthy & 0.02 \\
\hline & Sadness & IBD $>$ healthy & 0.03 \\
\hline SLS & Satisfaction with Life Scale & Healthy $>$ severe & 0.01 \\
\hline
\end{tabular}

G1, IBD patients; G2, healthy group; IBD, inflammatory bowel disease; TAQ, Buss-Perry Aggression Questionnaire; USAQ, Unconditional Self-Acceptance Questionnaire; GABS, General Attitudes and Beliefs Scale; PAD, Profile of Affective Distress; SLS, Satisfaction with Life Scale.

were obtained when comparing patients with regard to the presence/absence of symptoms like abdominal pain, articular pain or fatigue.

Patients who experience fatigue tend to have higher levels of self-downing $(\mathrm{P}=0.05)$, higher levels of need for comfort, $(\mathrm{P}=0.01<0.05)$, a higher demand for fairness $(\mathrm{P}=0.004<0.01)$, and also seem to have an overall higher level of irrationality, compared to patients who do not report the presence of this symptom $(\mathrm{P}=0.004<0.01)$. In the same time, fatigue accompanying the disease since its debut, make patients to express higher levels of need for accomplishment $(\mathrm{P}=0.02<0.05)$. No other significant differences were obtained for this symptom.

There are no significant differences regarding the levels of need for approval within the participants when considering health status, type of disease, duration of the disease, severity of the disease, presence/absence of main symptoms. G2 is more rational when compared to $\mathrm{G} 1(\mathrm{P}=0.05)$. Patients who experience abdominal pain have higher levels of rationality $(\mathrm{P}=0.02<0.05)$. No significant differences were obtained depending on duration of the disease, type or severity of disease, presence/absence of arthralgia or fatigue.
$P A D$. Regarding the negative emotions, patients with IBD generally experience more negative emotions compared to healthy participants $(\mathrm{P}=0.02<0.05)$, and especially those with moderate forms $(\mathrm{P}=0.04<0.05)$. In terms of symptoms, fatigue and arthralgia conduct to higher levels of negative emotions $(\mathrm{P}=0.004<0.01)$, respectively $(\mathrm{P}=0.01<0.05)$.

Patients with IBD have higher levels of functional negative emotions in comparison with healthy participants $(\mathrm{P}=0.05)$. No further relevant differences were obtained when considering type of disease, years since diagnosis, presence of abdominal pain. Patients who experience fatigue or arthralgia since the debut of the disease also have higher levels of functional negative emotions than patients who do not $(\mathrm{P}=0.004<0.01)$, $\mathrm{P}=0.01<0.05)$.

Positive emotions are higher in G2 than in G1 $(\mathrm{P}=0.008<0.01)$. Patients with Crohn's disease experience the least positive emotions $(\mathrm{P}=0.02<0.05)$. There are no significant differences between $\mathrm{CD}$ and UC. When compared to healthy participants, IBD patients have significantly lower levels of positive emotions irrespective of the duration of the disease $(</>7$ years $)(\mathrm{P}=0.03<0.05)$ or $>7$ years ago $\mathrm{P}=0.03<0.05)$. 
Table III. Statistically significant results in G1.

\begin{tabular}{|c|c|c|c|}
\hline Questionnaire & $\begin{array}{c}\text { Investigated } \\
\text { characteristic/subscale }\end{array}$ & Compared groups & Significance \\
\hline \multirow[t]{11}{*}{ COPE } & \multirow[t]{3}{*}{ Emotional venting } & Form: moderate $>$ severe & 0.03 \\
\hline & & Presence $>$ absence of fatigue & 0.03 \\
\hline & & Presence $>$ absence of articular pain & 0.005 \\
\hline & \multirow[t]{2}{*}{ Positive reinterpretation } & Form: moderate $>$ severe & 0.04 \\
\hline & & \multirow[t]{4}{*}{ Presence $>$ absence of abdominal pain } & 0.02 \\
\hline & Active coping & & 0.03 \\
\hline & Search for social support & & \\
\hline & for instrumental reasons & & 0.03 \\
\hline & Acceptance & \multirow[t]{3}{*}{ Presence $>$ absence of fatigue } & 0.02 \\
\hline & Behavioural disengagement & & 0.01 \\
\hline & Mental disengagement & & 0.01 \\
\hline USAQ & Unconditional self-acceptance & Moderate $>$ severe & 0.01 \\
\hline \multirow[t]{17}{*}{ GABS } & Self-downing & Presence $>$ absence of fatigue & 0.05 \\
\hline & Need for comfort & & 0.01 \\
\hline & Need for accomplishment & & 0.02 \\
\hline & Demand for fairness & & 0.004 \\
\hline & Irrationality & & 0.004 \\
\hline & Rationality & Presence $>$ absence of abdominal pain & 0.02 \\
\hline & \multirow[t]{3}{*}{ Functional negative emotions } & Presence $>$ absence of articular pain & 0.01 \\
\hline & & Presence $>$ absence of fatigue & 0.004 \\
\hline & & Presence $>$ absence of articular pain & 0.01 \\
\hline & \multirow[t]{3}{*}{ Anxiety } & Presence $>$ absence of abdominal pain & 0.04 \\
\hline & & Presence $>$ absence of fatigue & 0.04 \\
\hline & & Presence $>$ absence of articular pain & 0.05 \\
\hline & Worry & Presence $>$ absence of fatigue & 0.006 \\
\hline & \multirow[t]{2}{*}{ Depression } & Presence $>$ absence of fatigue & 0.004 \\
\hline & & Presence $>$ absence of articular pain & 0.04 \\
\hline & \multirow[t]{2}{*}{ Sadness } & Presence $>$ absence of fatigue & 0.01 \\
\hline & & Presence $>$ absence of articular pain & 0.003 \\
\hline \multirow[t]{3}{*}{ IPQ } & Illness cyclicity & Moderate $>$ severe & 0.01 \\
\hline & Consequences & Presence $>$ absence of abdominal pain & 0.05 \\
\hline & Emotional representations & Presence $>$ absence of fatigue & 0.04 \\
\hline
\end{tabular}

G2, healthy group; USAQ, Unconditional Self-Acceptance Questionnaire; GABS, General Attitudes and Beliefs Scale; IPQ, Illness Perception Questionnaire.

Patients with moderate or severe forms of IBD have lower levels of positive emotions when compared to healthy participants $(\mathrm{P}=0.04<0.05 ; \mathrm{P}=0.04<0.05)$. There are no significant differences between patients with moderate and severe forms of IBD regarding the presence/absence of symptoms such as abdominal/arthralgia or fatigue.

Regarding the anxiety, patients with IBD are more anxious than healthy participants $(\mathrm{P}=0.01<0.05)$. More specifically, patients with UC have the highest levels of anxiety $(\mathrm{P}=0.02<0.05 ; \mathrm{P}=0.006<0.01)$. $\mathrm{G} 1$ has levels of anxiety compared to $\mathrm{G} 2$ irrespective of the duration of their disease. $(\mathrm{P}=0.05 ; \mathrm{P}=0.04 ; \mathrm{P}=0.05)$. Patients with moderate forms also tend to have higher levels of anxiety when compared $\mathrm{G} 2,(\mathrm{P}=0.02>0.05)$, however no significant differences were obtained when comparing patients with severe forms of IBD with either the healthy group or with patients with moderate forms. There are higher levels of anxiety within patients who experience either abdominal pain $(\mathrm{P}=0.04<0.05)$, fatigue $(\mathrm{P}=0.04<0.05)$ or arthralgia $(\mathrm{P}=0.05)$.

There are no significant differences regarding the levels of concern depending on health status, type of disease, severity of disease or years since diagnosis. Patients who experience either articular or abdominal pain do not worry more than those who do not have these symptoms, whereas patients who experience fatigue have higher levels of concern $(\mathrm{P}=0.006<0.01)$.

There are no significant differences regarding the levels of depression depending on health status, type of disease, severity 
of disease or years since diagnosis or presence of abdominal pain. However, there are higher levels of depression among patients who feel either fatigued $(\mathrm{P}=0.004<0.01)$ or whom experience articular pain $(\mathrm{P}=0.04<0.05)$.

Patients with IBD are sadder than healthy persons $(\mathrm{P}=0.03<0.05)$, however, no significant differences were observed depending on type of disease, severity of disease, years since diagnosis or presence of abdominal pain. Fatigued patients and patients with arthralgia also have higher levels of sadness, when compared to patients who do not experience these symptoms $(\mathrm{P}=0.01<0.05 ; \mathrm{P}=0.003<0.01)$.

SLS. Healthy participants have higher levels of life satisfaction when compared to patients with severe forms of IBD $(\mathrm{P}=0.05)$. Life satisfaction does not differ between the participants depending on duration of the disease, type of disease or presence of symptoms since onset (fatigue, abdominal/articular pain) $(\mathrm{P}=0.01<0.05 ; \mathrm{P}=0.005<0.01)$.

$I P Q$. Regarding the perception of illness, there are no differences between patients in terms of illness coherence, personal or treatment control, consequences, timeline, or emotional representations. There are no significant differences depending on type of illness and duration of the disease. Patients with moderate forms of IBD expect their illness to be more cyclic than patients with severe forms $(\mathrm{P}=0.01<0.05)$. When it comes to the presence of specific symptoms, patients who experience abdominal pain also evaluate their illness as being more cyclic $(\mathrm{P}=0.05)$, as do patients with fatigue $(\mathrm{P}=0.02<0.05)$. Patients with fatigue also expect the consequences of their disease to be more severe than patients who do not have this symptom $(\mathrm{P}=0.003<0.01)$. The expected emotional impact of the illness (emotional representations) are also higher amongst patients with fatigue $(\mathrm{P}=0.04<0.05)$. There are no significant differences regarding the illness perception depending on the presence of articular pain.

\section{Discussion}

Having a chronic illness with poorly identified causes and rather hard to predict outcomes that could include surgery and cancer, impacts the patient's psychological well-being negatively to pain $(16,17)$. In retrospect, it is hard to determine whether patients suffering with IBD have a personality structure prone to developing mental health problems, or if the disease and its consequences directly influence the probability of developing psychological/psychiatric disorders.

Part of the studies on IBD patients (that investigated their emotional profile) have discovered higher levels of depression and especially a greater prevalence of anxiety amongst patients, when compared to healthy participants (18). Other studies have suggested greater variance on the presence of emotional disorders, based on the activity or the severity of the disease (19). The presence of functional negative emotions (worry, sadness) and the levels of positive emotions has not been investigated so far. However, the present study reveals that healthy participants have higher levels of positive emotions at all levels, when compared to patients with IBD, regardless of the severity or the duration of the disease. As mentioned, although previous studies have shown a greater prevalence of anxiety in IBD patients, studies on depression revealed mixed results (19). Based on these findings, our research shows significantly higher levels of anxiety and sadness among IBD patients, but no significant differences in terms of depression, when compared to a healthy group. Although, the increased presence of the feelings of sadness does not represent in itself a mental disorder, it impacts negatively the quality of life and can lead to depressive symptoms in the long-term or in specific circumstances where the coping mechanisms are challenged (for example, an active episode of the disease), making them relevant to study/know when considering the psychological profile of patients. The presence of certain symptoms also seems to be relevant when investigating the emotional profile of IBD patients: Patients with abdominal pain have higher levels of anxiety; patients with arthralgia have higher levels of anxiety, sadness and depression; patients who experience fatigue, have higher levels of worry, anxiety, sadness and depression.

Comparison studies are scarce on the four aggression-related dimensions (physical, verbal, hostility, anger) (20) with impulsivity being the closest trait taken into account more frequently so far (21). The higher scores in hostility in patients with IBD, especially in those with moderate forms and with $>7$ years since diagnosis, indicate they have a higher tendency to negatively evaluate events and people, and more feelings of injustice, when compared to the healthy group. Despite these differences in the cognitive domain, no significant differences were obtained regarding the behavioural/emotional component of aggression.

The relationship between emotions and cognitions has been long studied in the last decades, but not when it comes to patients with IBD. Eight categories of specific concerns have been identified, namely loss of control and energy, body image, lack of information, isolation, not reaching full potential and feeling dirty (22). However, there are no studies investigating the general presence of irrational attitudes and beliefs specifically in IBD patients, despite of their known importance for our mental health. The current study reveals that IBD patients (especially those with moderate forms of disease) have lower levels of rationality and higher levels of others-downing, when compared to a healthy group. Comparisons between patients show differences when considering the presence of fatigue with higher levels of self-downing, need for comfort, need for accomplishment, demand for fairness and overall irrationality for patients who report this symptom. Surprisingly, patients with abdominal pain have higher rationality levels.

The quality of life has been one of the most studied aspects in IBD patients $(20,23,24)$, with results showing influence of the presence and severity of the disease on one's general wellbeing. Our results also confirm the severity of the disease as a predictor of an overall poorer satisfaction with life.

Another highly studied factor is the impact of the presence of the disease on the self-image, with as much as $84 \%$ of the patients in one study reporting it as a perceived stigma (25). Changes regarding self-esteem and self-image include embarrassment because of the symptoms, alteration of body image due to medication side-effects/surgical interventions and can lead to self-accusations and feelings of guilt $(26,27)$. Considering all this, the patients' ability to unconditionally accept himself becomes important for his state of well-being and mental health. In the current study, patients with moderate 
severity of the disease have higher levels of unconditional self-acceptance when compared to either healthy participants or with patients with more severe forms. Given the circumstances, this can be viewed as a healthy coping mechanism to the asperities of the disease.

Assessing illness perception in chronic patients can improve treatment adherence and help improve the overall case management. The illness perception refers to the mental image one has about the disease, in terms of causes, symptoms, consequences and personal control. In IBD, previous studies have shown that the activity of the disease has an impact on the perception of the disease (28). This study shows that the presence of certain symptoms is also relevant for how patients perceive their disease. For instance, patients with abdominal pain assess IBD as being more cyclical than patients who do not experience this symptom. The perceived illness cyclicity is also different based on the severity of the disease (patients with moderate forms evaluate the cyclicity as being higher when compared to patients with severe forms). Patients who experience fatigue also evaluate their disease as having more negative consequences and a higher emotional impact, than patients who do not experience this symptom. This is understandable as fatigue has crippling effects on one's social and work life, also impacting the general well-being $(29,30)$.

Conclusion, this study clearly indicates that the psychological factors implied and different characteristics of IBD play a relevant role in the way the patients having this type of pathology deal with their disease.

\section{Acknowledgements}

The authors express their gratitude to Professor Simona Trip, University of Oradea, for help in the process of collecting the data.

\section{Funding}

No funding was received.

\section{Availability of data and materials}

At the private medical offices where the data were collected.

\section{Authors' contributions}

TI, DM, CH, RB, NN and OF selected the patients, analyzed and interpreted the patient data regarding results of self-administered questionnaires. SB, DMT, OGB, and CD made substantial contributions to the conception of the work and interpretation of data; also, they drafted the manuscript and were major contributors in writing the manuscript. All authors read and approved the final manuscript to be published. All the authors agreed to be accountable for all aspects of the work in ensuring that questions related to the accuracy or integrity of any part of the work are appropriately investigated and resolved.

\section{Ethics approval and consent to participate}

The research was performed according the WMA Declaration of Ethics Helsinki Medical Research Involving Human
Principles for Subjects and was approved by the Ethics Committee of the Clinical County Emergency Hospital of Oradea (Romania). All the subjects signed a written consent giving their acceptance to participate in the study.

\section{Patient consent for publication}

Not applicable.

\section{Competing interests}

The authors declare that they have no competing interests.

\section{References}

1. Burisch J, Jess T, Martinato M and Lakatos PL: The burden of inflammatory bowel disease in Europe. J Crohn's Colitis 7: 322-337, 2013.

2. Ng SC, Shi HY,Hamidi N, Underwood FE, Tang W, Benchimol EI, Panaccione R, Ghosh S, Wu JC, Chan FK, et al: Worldwide incidence and prevalence of inflammatory bowel disease in the 21st century: A systematic review of population-based studies. Lancet 390: 2769-2778, 2018.

3. Gheorghe C, Pascu O, Gheorghe L, Iacob R, Dumitru E, Tantau M, Vadan R, Goldis A, Balan G, Iacob S, et al: Epidemiology of inflammatory bowel disease in adults who refer to gastroenterology care in Romania: A multicentre study. Eur J Gastroenterol Hepatol 16: 1153-1159, 2004.

4. Mocanu D, Catuneanu AM, Diculescu M, Gologan S and Sporea I: Current epidemiologic trends in Crohn's disease: Data from a tertiary refferal centre in Bucharest. Maedica (Buchar) 5: 95-101, 2010.

5. Szigethy EM, Allen JI, Reiss M, Cohen W, Perera LP, Brillstein L, Cross RK, Schwartz DA, Kosinski LR, Colton JB, et al: The impact of mental and psychosocial factors on the care of patients with inflammatory bowel disease. Clin Gastroenterol Hepatol 15: 986-997, 2017.

6. Buss AH and Perry M: The aggression questionnaire. J Pers Soc Psychol 63: 452-459, 1992.

7. Carver CS, Scheier MF and Weintraub JK: Assessing coping strategies: A theoretically based approach. J Pers Soc Psychol 56: 267-283, 1989.

8. Chamberlain JM and Haaga DAF: Unconditional self-acceptance and psychological health. J Ration Emot Cogn Behav Ther 19: 163-176, 2001.

9. Flett G, Besser A, Davis RA and Hewit P: Dimensions of perfectionism, unconditional self-acceptance, and depression. J Ration Emot Cogn Behav Ther 21: 119-138, 2003.

10. Shepard L: Self-acceptance: The evaluative component of the self-concept construct. Am Ed Res J 16: 139-160, 1979.

11. Lindner H, Kirkby R, Wertheim F and Birch P: A brief assessment of irrational thinking: The Shortened General Attitude and Belief Scale. Cognit Ther Res 23: 651-663, 1999.

12. Ellis A and Bernard ME (eds): What is rational-emotive therapy? In: Clinical Applications of Rational-Emotive Therapy. PlenumPress, New York, NY, pp1-26, 1985.

13. Opris D and Macavei M: Profile of emotional distress. In: Clinical Evaluation System. David D (ed). RTS Publishing, Cluj-Napoca, 2007.

14. Moss-Morris R, Weinman J, Petrie KJ, Horne R, Cameron LD and Buick D: The revised illness perception questionnaire (IPQ-R). Psychol Health 17: 1-16, 2002.

15. Weinman J, Petrie K, Sharpe N and Walker S: Causal attributions in patients and spouses following a heart attack and subsequent lifestyle changes. Br J Health Psychol 5: 263-273, 2000.

16. Abdel-Daim MM, El-Tawil OS, Bungau SG and Atanasaov AG: Applications of antioxidants in metabolic disorders and degenerative diseases: Mechanistic approach. Oxid Med Cell Longev 2019: 4179676, 2019.

17. Pallag A, Roşca E, ţiŢ DM, MuŢiu G, Bungău SG and Pop OL: Monitoring the effects of treatment in colon cancer cells using immunohistochemical and histoenzymatic techniques. Rom J Morphol Embryol 56: 1103-1109, 2015. 
18. Ben Thabet J, Charfi N, Mnif L, Yaich S, Zouari L, Zouari N, Tahri N, Damak J and Maalej M: Emotional disorders and inflammatory bowel disease. Tunis Med 90: 557-563, 2012 (In French).

19. Byrne G, Rosenfeld G, Leung Y, Qian H, Raudzus J, Nunez C and Bressler B: Prevalence of anxiety and depression in patients with inflammatory bowel disease. Can J Gastroenterol Hepatol 2017: 6496727, 2017

20. Boye B, Lundin KE, Leganger S, Mokleby K, Jantschek G, Jantschek I, Kunzendorf S, Benninghoven D, Sharpe M, Wilhelmsen I, et al: The INSPIRE study: Do personality traits predict general quality of life (short form-36) in distressed patients with ulcerative colitis and Crohn's disease? Scand J Gastroenterol 43: 1505-1513, 2008.

21. La Barbera D, Bonanno B, Rumeo MV, Alabastro V, Frenda M, Massihnia E, Morgante MC, Sideli L, Craxì A, Cappello M, et al: Alexithymia and personality traits of patients with inflammatory bowel disease. Sci Rep 7: 41786, 2017.

22. Casati J, Toner BB, de Rooy EC, Drossman DA and Maunder RG: Concerns of patients with inflammatory bowel disease: A review of emerging themes. Dig Dis Sci 45: 26-31, 2000.

23. Faust AH, Halpern LF, Danoff-Burg S and Cross RK: Psychosocial factors contributing to inflammatory bowel disease activity and health-related quality of life. Gastroenterol Hepatol (NY) 8: 173-181, 2012.

24. Habibi F, Habibi ME, Gharavinia A, Mahdavi SB, Akbarpour MJ, Baghaei A and Emami MH: Quality of life in inflammatory bowel disease patients: A cross-sectional study. J Res Med Sci 22: 104, 2017.
25. Taft TH, Keefer L, Leonhard C and Nealon-Woods M: Impact of perceived stigma on inflammatory bowel disease patient outcomes. Inflamm Bowel Dis 15: 1224-1232, 2009.

26. Sammut J, Scerri J and Xuereb RB: The lived experience of adults with ulcerative colitis. J Clin Nurs 24: 2659-2667, 2015.

27. Woodward S, Dibley L, Coombes S, Bellamy A, Clark C, Czuber-Dochan W, Everelm L, Kutner S, Sturt J and Norton C: Identifying disease-specific distress in patients with inflammatory bowel disease. Br J Nurs 25: 649-660, 2016.

28. Vegni E, Gilardi D, Bonovas S, Corrò BE, Menichetti J, Leone D, Mariangela A, Furfaro F, Danese S and Fiorino G: Illness perception in inflammatory bowel disease patients is different between patients with active disease or in remission: A prospective cohort study. J Crohn's Colitis 13: 417-423, 2019.

29. Draghici T, Negreanu L, Bratu OG, Stoian AP, Socea B, Neagu TP, Stanescu AM, Manuc D and Diaconu CC: Paraneoplastic syndromes in digestive tumors: A review. Rom Biotechnol Lett 24: 813-819, 2019.

30. Manea M, Marcu D, Motofei I, Socea B, Stoian AP, Bratu OG, Gaman MA, Gaman AM, Stanescu AM and Diaconu C: Cardiovascular risk in patients with inflammatory bowel diseases: A review. Rom Biotechnol Lett 24: 366-373, 2019. 